\title{
Effect of inter-edge Coulomb interactions on transport through a point contact in a $\nu=5 / 2$ quantum Hall state
}

\author{
Sourin Das ${ }^{1,2}$, Sumathi RaO ${ }^{3}$ and Diptiman Sen 4 \\ 1 Institut für Festkörper-Forschung - Theorie 3, Forschungszentrum Jülich, 52425 Jülich, Germany \\ 2 Institut für Theoretische Physik A, RWTH Aachen, 52056 Aachen, Germany \\ ' 3 Harish-Chandra Research Institute, Chhatnag Road, Jhusi, Allahabad 211 019, India \\ 4 Centre for High Energy Physics, Indian Institute of Science, Bangalore 560 012, India \\ E-mail: s.das@fz-juelich.de; sumathi@hri.res.in; diptiman@cts.iisc.ernet.in
}

PACS 73.43.-f - Quantum Hall effects

PACS 73.43. Jn - Tunneling

PACS 71.10.Pm - Fermions in reduced dimensions (anyons, composite fermions, Luttinger liquid, etc.

\begin{abstract}
We study transport across a point contact separating two line junctions in a $\nu=5 / 2$ quantum Hall system. We analyze the effect of inter-edge Coulomb interactions between the chiral bosonic edge modes of the half-filled Landau level (assuming a Pfaffian wave function for the halffilled state) and of the two fully filled Landau levels. In the presence of inter-edge Coulomb interactions between all the six edges participating in the line junction, we show that the stable fixed point corresponds to a point contact which is neither fully opaque nor fully transparent. Remarkably, this fixed point represents a situation where the half-filled level is fully transmitting, while the two filled levels are completely backscattered; hence the fixed point Hall conductance is given by $G_{H}=\frac{1}{2} e^{2} / h$. We predict the non-universal temperature power laws by which the system approaches the stable fixed point from the two unstable fixed points corresponding to the fully connected case $\left(G_{H}=\frac{5}{2} e^{2} / h\right)$ and the fully disconnected case $\left(G_{H}=0\right)$.
\end{abstract}

In the last few years, there has been an upsurge in re' search on quantum Hall $(\mathrm{QH})$ systems in the context of non-abelian QH states, primarily because they open up unprecedented possibilities for realizing robust topological quantum computers [1]. One of the most promising candidates for the experimental realization of a non-abelian QH state is the $\nu=5 / 2$ state which was initially believed to be described by a Pfaffian (Pf) state [2], but now has a few other states [3] as plausible competitors.

Amongst the possible non-abelian QH states corresponding to various filling fractions, the $\nu=5 / 2$ state is relatively easier to access in an experiment. Hence, most theoretical proposals and experimental attempts related to probing of the non-abelian $\mathrm{QH}$ states focus on the $\nu=5 / 2$ state, and they are primarily related to transport measurements on the edge states. A complication that arises while making measurements on edge state corresponding to the half-filled Landau level ( $h a l f$-edge), which hosts the non-abelian quasi-particle, is that it is always masked by the two edge states corresponding to the two filled Landau levels. This is quite unlike the edge states of any fractional QH state in the first Landau level, for example, the $\nu=1 / 3$ state. So conductance measurements on such states will be in general difficult as far as directly probing them is concerned.

In this Letter, we propose a set-up involving a point contact embedded in a line junction which naturally gets rid of the two filled Landau levels by fully backscattering them at the point contact, and lets the half-edge transmit perfectly, without the need for fine-tuning of any gate voltage controlling transport across the point contact. When inter-edge interactions are introduced in the point contact geometry, the renormalization group flow automatically takes the system to this situation, which turns out to be the stable fixed point of the interacting edge theory.

Another motivation for studying the point contact geometry with inter-edge Coulomb interactions comes from the experiments of Dolev et al. [4] and Radu et al. [5]. The shot noise measurements by Dolev et al. confirmed the charge of the quasi-particle excitation in the half-filled 
Landau level in the $\nu=5 / 2$ state to be $e / 4$. This measurement is unlikely to get influenced by the presence of interedge Coulomb interaction. But, at the same time, this measurement by itself does not confirm the non-abelian nature of the state. On the other hand, the experiment by Radu et al. attempted to measure the power law scaling exponents associated with quasi-particle tunneling across a point contact. This measurement accompanied by the previous one on the quasi-particle charge can definitely help to decide which of the proposed candidate wave functions actually describe the $\nu=5 / 2$ state $\mathrm{QH}$ state. But the problem is that the power law scaling exponents can get influenced by the inter-edge Coulomb interactions, leading to non-universal values. Indeed, the experiment by Radu et al. observed non-universal values for the power laws. One of the prime reasons for such non-universal behavior could lie in the details of the inter-edge Coulomb interaction. Hence, this aspect of the problem is worth exploring. In general, the effects of inter-edge Coulomb interactions on the transport in edge states across a point contact have been studied in the past and have been shown to be non-trivial $[6,7]$.

We will now study the conductance across a point contact in the presence of inter-edge Coulomb interactions between both co-propagating and counter propagating edge modes of the half-filled Landau level (assuming a Pf wave function [8]) and the two fully filled Landau levels (assuming a chiral bosonic description for the edges), within a sharp edge scenario (i.e., with no edge reconstruction). Since a line junction is made by using metallic gates, the long-range part of the Coulomb interaction is screened. We will therefore model the Coulomb potential by a shortrange density-density interaction. As mentioned above, for weak inter-edge Coulomb interactions, we will show that the stable fixed point corresponds to an intermediate state in which the edge modes of the fully filled Landau levels are completely backscattered, while the edge corresponding to the half-filled Landau levels is completely transmitting at the point contact. This results in a fixed point conductance of $e^{2} /(2 h)$ in the low temperature limit. This situation is to be contrasted with the set-up where a point contact is pinched off in a $\mathrm{QH}$ liquid with a single edge state, for example the $\nu=1 / 3 \mathrm{QH}$ state [9]. Here it is known that there are only two fixed points (FPs), one corresponding to a perfectly transmitting point contact which is unstable, and the other corresponding to a completely opaque point contact which is stable (for weak inter-edge interactions). One can invert the stability of the two FPs by introducing strong enough inter-edge interactions in a line junction geometry [9], but no fixed point with intermediate transmission emerges in the theory. But in the case of the Pf edge, because of the multiple edge structure, there are fixed points which are neither fully transmitting nor fully opaque. Once we introduce even weak inter-edge Coulomb interaction, remarkably one such fixed point with intermediate transmission and reflection becomes the stable one.
In our theoretical model each edge of a $\nu=5 / 2 \mathrm{QH}$ state consists of an edge corresponding to $\nu=1 / 2$ and, say, spin $\uparrow$, an edge with $\nu=1$ and spin $\downarrow$, and an edge with $\nu=1$ and spin $\uparrow$ as we move outwards starting from the bulk. [This spatial ordering of the edges follows from the decrease in density on moving from the bulk towards the edge, and from the Zeeman splitting due to the coupling of the electron spin to the magnetic field.] The edge corresponding to $\nu=1 / 2$ is described by a charged chiral boson $\phi_{1}$ and a neutral (Majorana) fermion $\xi$ which has scaling dimension 1/2; in addition, an Ising spin field $\sigma$ with scaling dimension $1 / 16$ is required to describe the quasiparticles with charge $e / 4[8]$. The electron annihilation operator on the $\nu=1 / 2$ edge is given by $\psi_{e, 1}=\xi e^{i \sqrt{2} \phi_{1}}$ and has scaling dimension $1 / 2+1=3 / 2$ in the absence of interactions. The quasi-particle annihilation operator on the $\nu=1 / 2$ edge is given by $\psi_{q p, 1}=\sigma e^{i \phi_{1} /(2 \sqrt{2})}$, and has scaling dimension $1 / 16+1 / 16=1 / 8$ in the absence of interactions. The two edges with $\nu=1$ can be written in terms of charged chiral bosons $\phi_{2}$ and $\phi_{3}$. The electron operators on these edges are given by $\psi_{e, a}=e^{i \phi_{a}}$ $(a=2,3)$, and both their scaling dimensions are $1 / 2$ in the absence of interactions. Since the Majorana fermion $\xi$ and the Ising spin $\sigma$ are neutral, their scaling dimensions will not change when we introduce Coulomb interactions between the charged fields. However, the scaling dimensions of operators like $e^{i q_{a} \phi_{a}}$ depend on the strength of the interactions between counter propagating fields and will change when Coulomb interactions are introduced.

We now consider what happens if a $\nu=5 / 2 \mathrm{QH}$ liquid is split into two by applying a gate voltage along two semiinfinite lines with a gap (point contact) separating them; this depletes the electron density along those regions which are called line junctions. We denote the six edges from top to bottom of a $\nu=5 / 2$ edge with a line junction as $1(\nu=1 / 2$ edge, spin $\uparrow), 2(\nu=1$, spin $\downarrow), 3(\nu=1$, spin $\uparrow), 4(\nu=1$, spin $\uparrow), 5(\nu=1$, spin $\downarrow)$, and $6(\nu=$ $1 / 2$, spin $\uparrow)$; the edges of the other $\nu=5 / 2$ edge with a line junction will be similarly denoted as $1^{\prime}, 2^{\prime}, \cdots, 6^{\prime}$. The different edges of the two line junctions can now be connected to each other in several ways. Assuming that only edges with the same spin can connect to each other and that there are no crossings between different edges, there are four possible configurations as shown in Figs. 1 (a-d); all of them represent fixed points in the theory. They correspond to the following boundary conditions at the location of the point contact, called $x=0$, between the different bosonic fields: (a) $\phi_{a}=\phi_{a^{\prime}}$ for $a=1,2, \cdots, 6$, (b) $\phi_{a}=\phi_{a^{\prime}}$ for $a=1,2,5,6, \quad \phi_{3}=\phi_{4}$, and $\phi_{3^{\prime}}=\phi_{4^{\prime}}$, (c) $\phi_{1}=\phi_{1^{\prime}}, \phi_{6}=\phi_{6^{\prime}}, \phi_{2}=\phi_{5}, \phi_{3}=\phi_{4}, \phi_{2^{\prime}}=\phi_{5^{\prime}}$, and $\phi_{3^{\prime}}=\phi_{4^{\prime}}$, and (d) $\phi_{1}=\phi_{6}, \phi_{2}=\phi_{5}, \phi_{3}=\phi_{4}, \phi_{1^{\prime}}=$ $\phi_{6^{\prime}}, \phi_{2^{\prime}}=\phi_{5^{\prime}}$, and $\phi_{3^{\prime}}=\phi_{4^{\prime}}$. These conditions can be written in terms of a current splitting matrix $\mathcal{M}$ as follows. We define two vectors $\phi_{I}$ and $\phi_{O}$, denoting incoming and outgoing fields, as $\phi_{i I}=\left(\begin{array}{llllll}\phi_{1} & \phi_{2} & \phi_{3} & \phi_{6^{\prime}} & \phi_{5^{\prime}} & \phi_{4^{\prime}}\end{array}\right)$, and

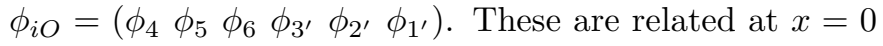




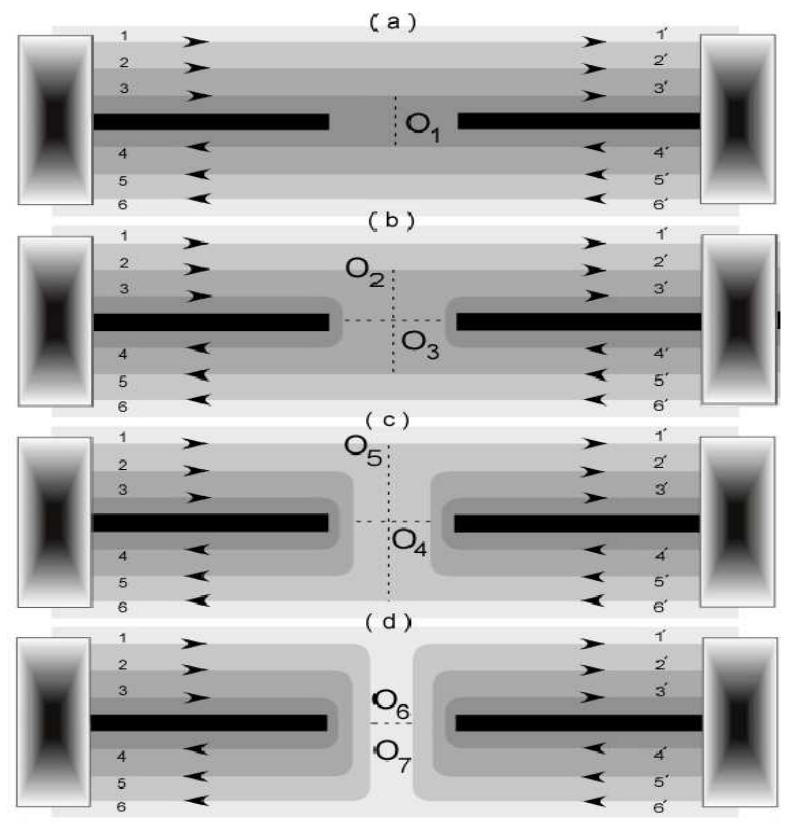

Fig. 1: Pictures of different configurations of two $\nu=5 / 2$ edges split by line junctions which are themselves separated by a point contact in the middle $(x=0)$. The six edges (three incoming and three outgoing) are marked as $1-6$ on the left and $1^{\prime}-6^{\prime}$ on the right; they are connected to each other at $x=0$ in four possible ways shown in (a-d). The black regions denote the line junctions; the other shades show the filling fractions $0,1,2$ and $5 / 2$ from darkest to lightest, the white regions denoting the $\nu=5 / 2$ bulk.

by a $6 \times 6$ real orthogonal matrix $\mathcal{M}$ as $\phi_{i O}=\sum_{j} \mathcal{M}_{i j} \phi_{j I}$. Current conservation implies that each column of $\mathcal{M}$ must add up to 1.

We will study the stability of the configurations in Fig. 1 with respect to tunneling between different edges. To do this, we will consider the following tunneling operators at the point $x=0$ between nearby edges with the same spin in the configurations shown in Figs. 1 (a-d):

(a) $O_{1}=\psi_{e, 4}^{\dagger} \psi_{e, 3}=\exp \left[i\left(\phi_{3}-\phi_{4}\right)\right]$,

(b) $O_{2}=\psi_{e, 5}^{\dagger} \psi_{e, 2}=\exp \left[i\left(\phi_{2}-\phi_{5}\right)\right]$, and $O_{3}=\psi_{e, 3^{\prime}}^{\dagger} \psi_{e, 3}=$ $\exp \left[i\left(\phi_{3}-\phi_{3^{\prime}}\right)\right]$,

(c) $O_{4}=\psi_{e, 2^{\prime}}^{\dagger} \psi_{e, 2}=\exp \left[i\left(\phi_{2}-\phi_{2^{\prime}}\right)\right]$, and $O_{5}=\psi_{e, 6}^{\dagger} \psi_{e, 1}=$ $\xi_{1} \xi_{6} \exp \left[i \sqrt{2}\left(\phi_{1}-\phi_{6}\right)\right]$,

(d) $O_{6}=\psi_{e, 1^{\prime}}^{\dagger} \psi_{e, 1}=\xi_{1} \xi_{1^{\prime}} \exp \left[i \sqrt{2}\left(\phi_{1}-\phi_{1^{\prime}}\right)\right]$, and $O_{7}=$ $\psi_{q p, 1^{\prime}}^{\dagger} \psi_{q p, 1}=\sigma_{1} \sigma_{1^{\prime}} \exp \left[i\left(\phi_{1}-\phi_{1^{\prime}}\right) /(2 \sqrt{2})\right]$.

Of these seven operators, $O_{1}-O_{6}$ correspond to electron tunneling, while $O_{7}$ corresponds to quasi-particle tunneling. In the absence of interactions, we can easily compute the scaling dimensions $d_{i}$ of the operators $O_{i}$. We find that $d_{1}=d_{2}=d_{3}=d_{4}=1$ (marginal), $d_{5}=d_{6}=3$ (irrelevant), and $d_{7}=1 / 4$ (relevant). For weak interactions, $O_{5}$ and $O_{6}\left(O_{7}\right)$ will continue to remain irrelevant (relevant); thus Fig. 1 (d) is an unstable FP. We will study below whether the operators $O_{1}-O_{4}$ will become relevant or irrelevant for weak interactions. We note that if there are density-density interactions only between copropagating modes, then none of the scaling dimensions would get modified. It is therefore important to consider interactions between counter propagating modes as well.

In any of the configurations shown in Fig. 1, there are three right moving and three left moving bosonic fields either far to the left or far to the right of the point contact. Ignoring the two Majorana fermion fields, the Lagrangian density of the six bosonic fields can be written as

$$
\mathcal{L}=-\frac{1}{4 \pi}\left(\sum_{a=1}^{6} \epsilon_{a} \partial_{t} \phi_{a} \partial_{x} \phi_{a}+\sum_{a, b=1}^{6} \partial_{x} \phi_{a} K_{a b} \partial_{x} \phi_{b}\right)
$$

where $\epsilon_{a}=1(-1)$ for the three right (left) moving fields respectively. In Eq. (11), we have absorbed the velocities in the diagonal elements $K_{a a}$ for simplicity; three of the velocities will be positive and three negative. [For repulsive interactions between edges $a$ and $b$, we have $\epsilon_{a} \epsilon_{b} K_{a b}>0$ ]. The densities and currents are given by $\rho_{a}=\left(\epsilon_{a} / 2 \pi\right) \partial_{x} \phi_{a}$ and $j_{a}=-\left(\epsilon_{a} / 2 \pi\right) \partial_{t} \phi_{a}$; these satisfy the equations of continuity. To quantize the theory, we impose the equaltime commutation relations $\left[\phi_{a}(x), \rho_{b}(y)\right]=-i \delta_{a b} \delta(x-y)$. These will be satisfied if the fields are given at time $t=0$ by $\phi_{a}(x)=\int_{0}^{\infty} \frac{d k}{k}\left[c_{a k} e^{i \epsilon_{a} k x}+c_{a k}^{\dagger} e^{-i \epsilon_{a} k x}\right]$, where $\left[c_{a k}, c_{b k^{\prime}}^{\dagger}\right]=\delta_{a b} k \delta\left(k-k^{\prime}\right)$.

The Lagrangian in Eq. (11) can be diagonalized either by a Bogoliubov transformation [10] or, equivalently, by solving the equations of motion. We assume that the fields take the form $\phi_{a}=X_{a \alpha} e^{i k\left(x-\tilde{v}_{\alpha} t\right)}$, where the index $\alpha$ (= $1,2, \cdots, 6)$ labels the different solutions, and $\tilde{v}_{\alpha}$ are the corresponding velocities. The $X_{a \alpha}$ (which are real) and the $\tilde{v}_{\alpha}$ can be obtained by solving the equations

$$
\sum_{b=1}^{6} \epsilon_{a} K_{a b} X_{b \alpha}=\tilde{v}_{\alpha} X_{a \alpha}
$$

If the problem is well defined (namely, the interactions between the counter propagating modes are not too strong compared to the interactions between co-propagating modes), the velocities $\tilde{v}_{\alpha}$ will all be real, with three of them being positive (right moving) and three being negative (left moving). We introduce a label $\epsilon_{\alpha}=1$ and -1 for the eigenmodes with $\tilde{v}_{\alpha}>0$ and $<0$ respectively. The eigenvectors can then be normalized so that

$$
\begin{aligned}
& \sum_{a=1}^{6} \epsilon_{a} \epsilon_{\alpha} X_{a \alpha} X_{a \beta}=\delta_{\alpha \beta} \\
& \sum_{\alpha=1}^{6} \epsilon_{a} \epsilon_{\alpha} X_{a \alpha} X_{b \alpha}=\delta_{a b}
\end{aligned}
$$

We introduce the projection operators $P_{a \alpha, \pm}=(1 \pm$ $\left.\epsilon_{a} \epsilon_{\alpha}\right) / 2$. The original and new (Bogoliubov transformed) 
annihilation operators $c_{a k}$ and $\tilde{c}_{\alpha k}$ are related as

$$
\begin{aligned}
\tilde{c}_{\alpha k} & =\sum_{a=1}^{6} X_{a \alpha}\left[P_{a \alpha,+} c_{a k}-P_{a \alpha,-} c_{a k}^{\dagger}\right], \\
c_{a k} & =\sum_{\alpha=1}^{6} X_{a \alpha}\left[P_{a \alpha,+} \tilde{c}_{\alpha k}+P_{a \alpha,-} \tilde{c}_{\alpha k}^{\dagger}\right] .
\end{aligned}
$$

Using Eq. (3), we can verify that $\left[c_{a k}, c_{b k^{\prime}}\right]=0$ and $\left[c_{a k}, c_{b k^{\prime}}^{\dagger}\right]=\delta_{a b} k \delta\left(k-k^{\prime}\right)$ imply that $\left[\tilde{c}_{\alpha k}, \tilde{c}_{\beta k^{\prime}}\right]=0$ and $\left[\tilde{c}_{\alpha k}, \tilde{c}_{\beta k^{\prime}}^{\dagger}\right]=\delta_{\alpha \beta} k \delta\left(k-k^{\prime}\right)$. Eq. (4) implies that the original and new bosonic fields are related at the point $x=0$ as $\tilde{\phi}_{\alpha}=\sum_{a=1}^{6} \epsilon_{a} \epsilon_{\alpha} X_{a \alpha} \phi_{a}$ and $\phi_{a}=\sum_{\alpha=1}^{6} X_{a \alpha} \tilde{\phi}_{\alpha}$.

We will choose the same values of the parameters $K_{a b}$ for the six edges on the left and on the right of the configurations in Fig. 1; this ensures that the same matrix $X_{a \alpha}$ will govern the fields on both sides of the point contact, $\phi_{a}, \tilde{\phi}_{\alpha}$ and $\phi_{a^{\prime}}, \tilde{\phi}_{\alpha^{\prime}}$. Further, we will assume for simplicity that $K_{a b}$ is mirror symmetric under a reflection about the line junction, i.e., $K_{a, b}=K_{7-a, 7-b}$ for all values of $a$ and $b$. For any such choice of the parameters $K_{a b}$, we can compute the scaling dimensions of the tunneling operators $O_{1}-O_{7}$ mentioned earlier as follows. We first find the eigenvectors $X_{a \alpha}$ as indicated in Eqs. (2) and (3). We can then relate the Bogoliubov transformed incoming and outgoing fields to the original incoming and outgoing fields. These take the forms

$$
\begin{aligned}
\tilde{\phi}_{i I} & =\sum_{j=1}^{6}\left[A_{i j} \phi_{j I}-B_{i j} \phi_{j O}\right], \\
\tilde{\phi}_{i O} & =\sum_{j=1}^{6}\left[C_{i j} \phi_{j O}-D_{i j} \phi_{j I}\right],
\end{aligned}
$$

where the matrices $A-D$ can be constructed as follows. If the $6 \times 6$ matrix $X_{a \alpha}$ has a block form

$$
X=\left(\begin{array}{ll}
X_{1} & X_{2} \\
X_{3} & X_{4}
\end{array}\right),
$$

where the $X_{i}$ 's are $3 \times 3$ matrices, the matrices $A-D$ have the block forms

$$
\begin{aligned}
A=\left(\begin{array}{cc}
X_{1} & 0 \\
0 & X_{1}
\end{array}\right), & B=\left(\begin{array}{cc}
X_{2} & 0 \\
0 & X_{2}
\end{array}\right) \\
C=\left(\begin{array}{cc}
X_{4} & 0 \\
0 & X_{4}
\end{array}\right), & D=\left(\begin{array}{cc}
X_{3} & 0 \\
0 & X_{3}
\end{array}\right) .
\end{aligned}
$$

Since $\phi_{i O}$ and $\phi_{i I}$ are related at $x=0$ as $\phi_{O}=\mathcal{M} \phi_{I}$, Eq. (5) implies that $\phi_{I}=(A-B \mathcal{M})^{-1} \tilde{\phi}_{I}$ and $\phi_{O}=\mathcal{M}(A-$ $B \mathcal{M})^{-1} \tilde{\phi}_{I}$. This gives a relation between the twelve fields $\phi_{a}$ and $\phi_{a^{\prime}}$ and the six incoming Bogoliubov fields $\tilde{\phi}_{i I}$ which can be taken to be mutually independent. Since the scaling dimension of $\exp \left(i \sum_{i} q_{i} \tilde{\phi}_{i I}\right)$ is $(1 / 2) \sum_{i} q_{i}^{2}$, we can find the scaling dimension of the exponential of any linear combination of the fields $\phi_{a}$ and $\phi_{a^{\prime}}$.

The scaling dimensions of the operators $\mathrm{O}_{1}-\mathrm{O}_{7}$ can now be computed. The computation is somewhat involved for the configurations shown in Figs. 1 (b) and 1 (c), but is simple for Figs. 1 (a) and 1 (d). The scaling dimension of the operator $O_{1}=\exp \left[i\left(\phi_{3}-\phi_{4}\right)\right]$ in Fig. 1 (a) is given by $d_{1}=(1 / 2) \sum_{a=1}^{6}\left(X_{3 a}-X_{4 a}\right)^{2}$, while the scaling dimensions of the operators $O_{6}$ and $O_{7}$ in Fig. 1 (d) are given by $d_{6}=1+2 \gamma$ and $d_{7}=(1+\gamma) / 8$ respectively, where $\gamma=\left(X_{11}+X_{16}\right)^{2}+\left(X_{12}+X_{15}\right)^{2}+\left(X_{13}+X_{14}\right)^{2}$.

To be explicit, let us discuss a specific form of the interaction parameters given by the matrix $K_{a b}$. We take all the diagonal entries $K_{a a}$ equal to $v_{0}$, the off-diagonal entries corresponding to all pairs of co-propagating modes equal to $v_{1}$, and the off-diagonal entries corresponding to all pairs of counter propagating modes equal to $-v_{2}$, where the three parameters $v_{i}$ are all positive (corresponding to repulsive interactions). Namely, $K$ has the highly symmetric form

$$
K_{a b}=\left(\begin{array}{cccccc}
v_{0} & v_{1} & v_{1} & -v_{2} & -v_{2} & -v_{2} \\
v_{1} & v_{0} & v_{1} & -v_{2} & -v_{2} & -v_{2} \\
v_{1} & v_{1} & v_{0} & -v_{2} & -v_{2} & -v_{2} \\
-v_{2} & -v_{2} & -v_{2} & v_{0} & v_{1} & v_{1} \\
-v_{2} & -v_{2} & -v_{2} & v_{1} & v_{0} & v_{1} \\
-v_{2} & -v_{2} & -v_{2} & v_{1} & v_{1} & v_{0}
\end{array}\right) .
$$

We then find that the Bogoliubov transformation simplifies if we first define the linear combinations

$$
\begin{aligned}
\Phi_{1} & =\frac{1}{\sqrt{3}}\left(\phi_{1}+\phi_{2}+\phi_{3}\right), \\
\Phi_{2} & =\frac{1}{\sqrt{2}}\left(\phi_{1}-\phi_{2}\right), \\
\Phi_{3} & =\frac{1}{\sqrt{6}}\left(\phi_{1}+\phi_{2}-2 \phi_{3}\right), \\
\Phi_{4} & =\frac{1}{\sqrt{3}}\left(\phi_{6}+\phi_{5}+\phi_{4}\right), \\
\Phi_{5} & =\frac{1}{\sqrt{2}}\left(\phi_{6}-\phi_{5}\right), \\
\Phi_{6} & =\frac{1}{\sqrt{6}}\left(\phi_{6}+\phi_{5}-2 \phi_{4}\right) .
\end{aligned}
$$

The corresponding Bogoliubov transformed fields are $\tilde{\Phi}_{i}$. We find that $\tilde{\Phi}_{1}$ and $\tilde{\Phi}_{4}$ both have the velocity $\tilde{v}_{1}=$ $\sqrt{\left(v_{0}+2 v_{1}\right)^{2}-9 v_{2}^{2}}$, and they are related to the original fields $\Phi_{1}$ and $\Phi_{4}$ as

$$
\begin{aligned}
\tilde{\Phi}_{1} & =\cosh \theta \Phi_{1}-\sinh \theta \Phi_{4}, \\
\tilde{\Phi}_{4} & =\cosh \theta \Phi_{4}-\sinh \theta \Phi_{1}, \\
\text { where } e^{-2 \theta} & =\sqrt{\frac{v_{0}+2 v_{1}-3 v_{2}}{v_{0}+2 v_{1}+3 v_{2}}} .
\end{aligned}
$$

Note that $\theta>0$ if the interaction $v_{2}$ between counter propagating modes is repulsive. All the remaining fields $\Phi_{2,3,5,6}$ have the velocity $\tilde{v}_{2}=v_{0}-v_{1}$, and they are not affected by the Bogoliubov transformation. The validity of all these relations requires that $v_{0}>v_{1}$ and $v_{0}+2 v_{1}>3 v_{2}$. We then find that the scaling dimensions of the operators $O_{1}, O_{6}$ and $O_{7}$ are given by $d_{1}=\left(2+e^{-2 \theta}\right) / 3, d_{6}=$ 
$\left(7+2 e^{2 \theta}\right) / 3$, and $d_{7}=\left(5+e^{2 \theta}\right) / 24$. We see that $O_{1}$ is relevant if $\theta>0, O_{6}$ is always irrelevant, and $O_{7}$ is relevant as long as $e^{2 \theta}<19$, i.e., if the interaction $v_{2}$ is not very strong. The scaling dimensions of the other four operators $O_{2,3,4,5}$ can be found numerically as indicated above. We find that $O_{2}$ is relevant while $O_{3,4,5}$ are irrelevant for all values of $\theta>0$. This implies that the configuration in Fig. 1 (a) is unstable, 1 (d) is unstable if $v_{2}$ is not very large, 1 (b) is unstable, and 1 (c) is stable. In Table 1, we present the scaling dimensions of the seven operators for a number of values of $e^{-2 \theta}$ which is a measure of the strength of the interactions between the counter propagating modes.

\begin{tabular}{|c|c|c|c|c|c|}
\hline$e^{-2 \theta} \rightarrow$ & 0.2 & 0.4 & 0.6 & 0.8 & 1 \\
\hline$d_{1}$ & 0.73 & 0.80 & 0.87 & 0.93 & 1 \\
\hline$d_{2}$ & 0.64 & 0.75 & 0.85 & 0.93 & 1 \\
\hline$d_{3}$ & 1.36 & 1.25 & 1.15 & 1.07 & 1 \\
\hline$d_{4}$ & 1.57 & 1.33 & 1.18 & 1.08 & 1 \\
\hline$d_{5}$ & 1.86 & 2.33 & 2.64 & 2.85 & 3 \\
\hline$d_{6}$ & 5.67 & 4.00 & 3.44 & 3.17 & 3 \\
\hline$d_{7}$ & 0.42 & 0.31 & 0.28 & 0.26 & 0.25 \\
\hline
\end{tabular}

Table 1. Scaling dimensions $d_{i}$ of the seven operators for a number of values of $e^{-2 \theta} \leq 1$ (repulsive interactions).

We have numerically studied the effects of some other forms of the matrix $K_{a b}$. For a large range of interactions between all the edges, we find that the configurations in Figs. 1 (a), (b) and (d) are unstable to the tunnelings described by the operators $O_{1}, O_{2}$ and $O_{7}$, namely, their scaling dimensions $d_{1}, d_{2}$ and $d_{7}$ are all less than 1 . (The operators $O_{3}$ and $O_{6}$ are irrelevant). However, the configuration in Fig. 1 (c) is stable under the tunnelings described by both $O_{4}$ and $O_{5}$, namely, their scaling dimensions $d_{4}$ and $d_{5}$ are larger than 1 . These results may be expected since it is known that weak interactions make backscattering between two perfectly transmitting $\nu=1$ edges relevant, and tunneling between two perfectly reflecting $\nu=1$ edges irrelevant [11]. The stability of the configuration in Fig. 1 (c) has a remarkable consequence. If we measure the Hall conductance $G$ between the left and right halves of the system, only the $\nu=1 / 2$ mode would contribute since the $\nu=1$ modes are completely backscattered; we should therefore obtain a low-temperature value of $G$ equal to $e^{2} /(2 h)$. An experimental confirmation of this would provide the first example in a $\mathrm{QH}$ system of such a value of $G$.

Experimentally, two different possibilities exist depending on whether we start at high temperature with the configuration in Fig. 1 (a) (all modes fully transmitting) or 1 (d) (all modes fully backscattered). If we start with Fig. 1 (a), we have $G=5 e^{2} /(2 h)$ at high temperature. Then if switch on a small amount of backscattering at the point contact, then a RG argument implies that as the temperature $T$ is decreased, $G$ will decrease from $5 e^{2} /(2 h)$ as $1 / T^{1-d_{1}}$ (region (i) in Fig. 2). This will continue till $G$ flows at low temperature to $e^{2} /(2 h)$ as in Fig. 1 (c).

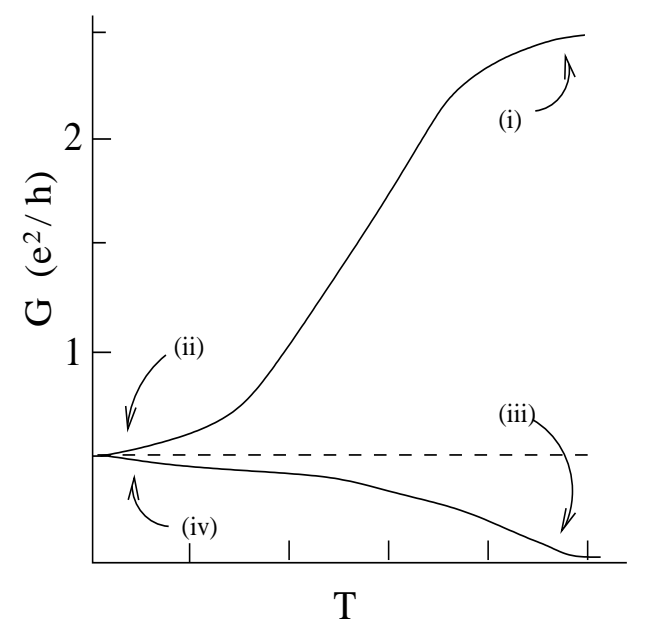

Fig. 2: Variation of Hall conductance $G$ (in units of $e^{2} / h$ ) with temperature $T$ (in arbitrary units) for two possible starting points at high temperature. The upper curve shows a RG flow from an unstable FP at $5 e^{2} /(2 h)$ to a stable $\mathrm{FP}$ at $e^{2} /(2 h)$ at low temperature, while the lower curve shows a flow from an unstable FP at 0 to $e^{2} /(2 h)$ at low temperature. The regions (i-iv) are explained in the text.

Also note that, at some intermediate temperature, the system will go through the configuration in Fig. 1 (b) where $G=3 e^{2} /(2 h)$.] As $T \rightarrow 0, G$ will approach $e^{2} /(2 h)$ from above as $T^{d_{4}-1}$ (region (ii) in Fig. 2), since this results from the vanishing of the irrelevant tunneling $\mathrm{O}_{4}$. On the other hand, if we start with Fig. 1 (d) at high temperature, $G$ is equal to 0 and then switch on a small amount of tunneling at the point contact; it will then increase as $1 / T^{1-d_{7}}$ (region (iii) in Fig. 2) as the temperature is reduced. Finally, it will approach $e^{2} /(2 h)$ from below as $T^{d_{5}-1}$ at low temperature (region (iv) in Fig. 2), since this follows from the vanishing of the irrelevant tunneling $O_{5}$. From an experimental point of view, it might be expected that the line junction formed by the gates would allow electrons to tunnel across it, leading to a disorder dominated phase. But it may be possible to avoid this by fine etching of the two-dimensional electron gas along the line junction beneath the gates. This should allow the scenario we have proposed to be experimentally accessible. Note that we have not considered the outer edges of the $\nu=5 / 2$ bulk which lie far away from the line junction as is depicted in Fig. 3. This would be justified in a transport measurement set-up in which the top, bottom and the left contacts are at the same voltage, and the bias is applied at the right contact (see Fig. 3). This will ensure that the current that is collected at the left contact is solely due to the transport taking place across the line junction and not due to the outer two edges, and hence can confirm our prediction.

To conclude, we have shown that for the $\nu=5 / 2$ state, the presence of inter-edge Coulomb interactions can lead to a stable fixed point with a fixed point conductance of 


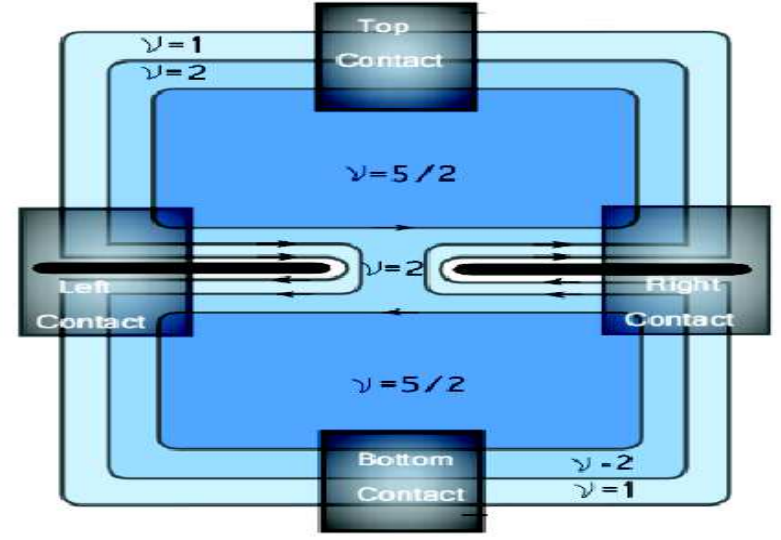

Fig. 3: Schematic picture of a four-terminal set-up which allows for the measurement of a conductance of $\frac{e^{2}}{2 h}$ arising solely due to transport across the line junction alone and not the outer two top and bottom, left and right moving edge states. The four gray patches represent the ohmic contacts. The deep blue region represents the $\nu=5 / 2$ Hall states, and the lighter shades represents the region of the other two integer filling fractions.

$G_{H}=e^{2} /(2 h)$, which uniquely identifies a Hall plateau with an effective filling fraction of half. We have predicted the power laws of the deviations of the conductance from its fixed point value, as functions of the interaction strengths between the various edge states. We have also provided an explicit experimental set-up which can check the predictions that we make in this letter. In general, the strategy outlined here can be applied to unmask any fractional Hall edge masked by integer Hall edge states.

SD thanks Moty Heiblum for discussions. SD and DS thank DST, India for financial support under Project No. SR/S2/CMP-27/2006.

\section{REFERENCES}

[1] M. H. Freedman, A. Kitaev, M. J. Larsen, and Z. Wang, Bull. Amer. Math. Soc. 40, 31 (2003).

[2] G. Moore and N. Read, Nucl. Phys. B 360, 362 (1991).

[3] N. Read and E. Rezayi, Phys. Rev. B 59, 8084 (1999); M. Levin, B. I. Halperin, and B. Rosenow, Phys. Rev. Lett. 99, 236806 (2007); S.-S. Lee, S. Ryu, C. Nayak, and M. P. A. Fisher, Phys. Rev. Lett. 99, 236807 (2007); B. J. Overbosch and X.-G. Wen, arXiv:0804.2087.

[4] M. Dolev, M. Heiblum, V. Umansky, A. Stern, and D. Mahalu, Nature 452, 829 (2008).

[5] I. P. Radu, J. B. Miller, C. M. Marcus, M. A. Kastner, L. N. Pfeiffer, and K. W. West, Science 320, 899 (2008).

[6] L. P. Pryadko, E. Shimshoni, and A. Auerbach, Phys. Rev. B 61, 10929 (2000).

[7] E. Papa and A. H. MacDonald, Phys. Rev. Lett. 93, 126801 (2004).

[8] P. Fendley, M. P. A. Fisher, and C. Nayak, Phys. Rev. B 75, 045317 (2007).

[9] C. L. Kane and M. P. A. Fisher, Phys. Rev. B 56, 15231 (1997).
[10] C. Tsallis, J. Math. Phys. 19, 277 (1978).

[11] C. L. Kane and M. P. A. Fisher, Phys. Rev. B 46, 15233 (1992). 